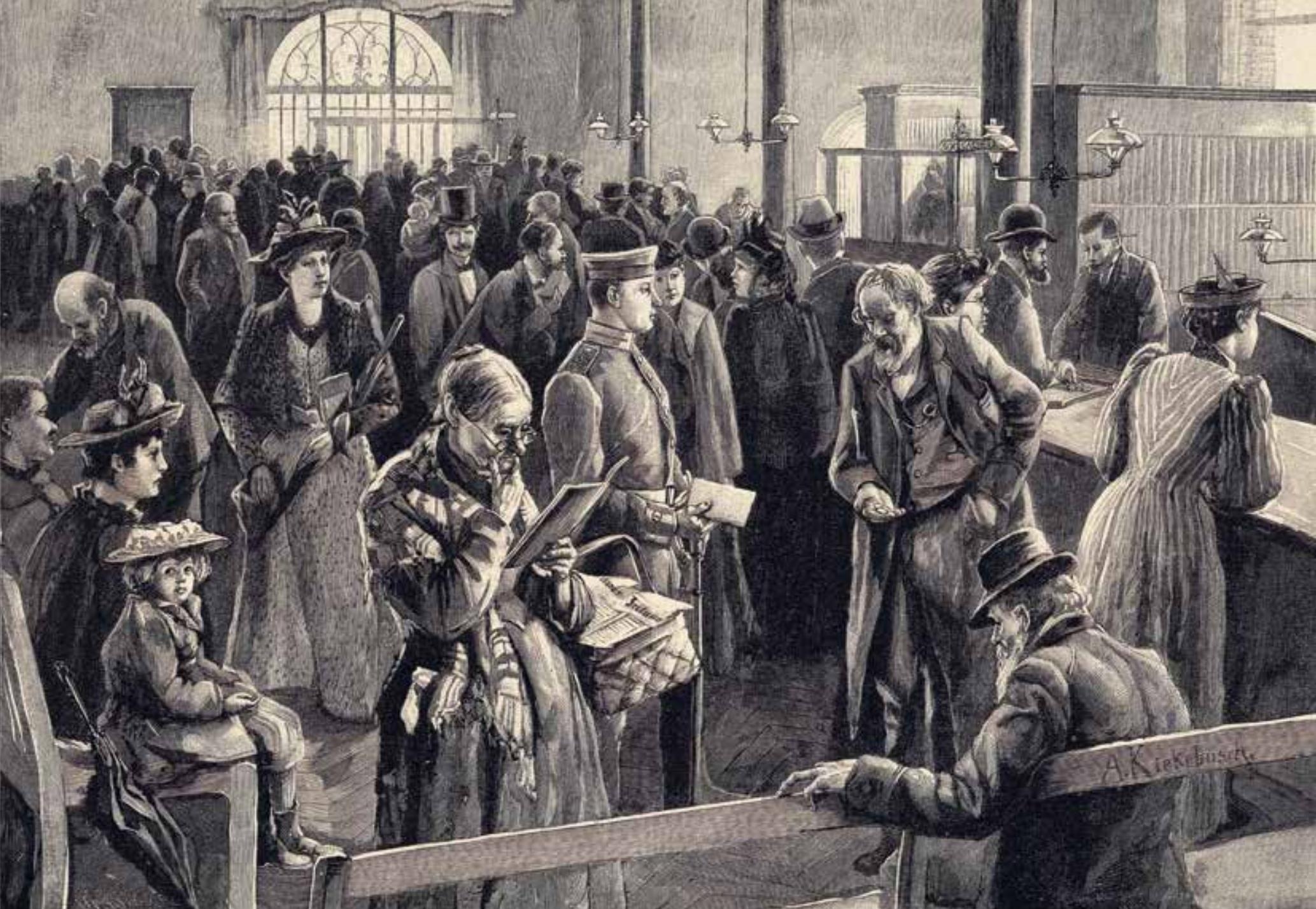

\title{
Die Verbreitung des Spar- und Sparkassengedankens in Deutschland im 18. und 19. Jahrhundert
}

Schalterraum der Berliner Sparkasse im Gebäude am Mühlendamm, Stich nach einer Zeichnung von Albert Kiekebusch, 1894

( ) Historisches Archiv des Ostdeutschen Sparkassenverbandes

Wiederabdruck des Aufsatzes aus Robert Muschalla (Hrsg.): Sparen. Geschichte einer deutschen Tugend. Berlin 2018, S. 31-42.

\section{Thorsten Wehber}

Institutionen zu schaffen, mit deren Hilfe Menschen Rücklagen für Notzeiten bilden können, ist eine sehr alte Idee. Pläne für solche Einrichtungen wurden bereits 1611 in Frankreich (Hugues Delestre: Le Premier Plant du Mont de Piété François consacré à Dieu) und 1697 in England (Daniel Defoe: An Essay Upon Projects) entwickelt. ${ }^{1}$ In Deutschland publizierte Johann Heinrich Gottlob von Justi 1761 in seinem Buch „Die Grundfeste zu der Macht und Glückseligkeit der Staaten“ den Gedanken, in jeder großen Stadt eine „Manufaktur-Armen-Casse“ einzurichten. In sie sollten alle Arbeiter wöchentlich eine kleine Geldsumme einzahlen. ${ }^{2}$
Diese Pläne entstanden vor dem Hintergrund, dass in der frühen Neuzeit stets große Teile der Bevölkerung arm oder armutsgefährdet waren. Missernten, Wirtschaftskrisen und die häufigen Kriege stellten die europäischen Gesellschaften immer wieder vor existenzielle Herausforderungen. Dass in der zweiten Hälfte des 18. Jahrhunderts ein starkes Bevölkerungswachstum einsetzte, verschärfte die Situation. Denn Landwirtschaft, Handel und Gewerbe konnten längst nicht allen Menschen ein stetiges und sicheres Einkommen geben.

Das 18. Jahrhundert war aber auch das Zeitalter der Aufklärung. Aufgeklärte Philosophen wie Im-
1 Vgl. Günter Ashauer: Von der Ersparungscasse zur Sparkassen-Finanzgruppe. Die deutsche Sparkassenorganisation in Geschichte und Gegenwart. Stuttgart 1991, S. $31 \mathrm{ff}$.

2 Martin Peters: Sparen - Leihen - Vorsorgen. Die Gemeinnützigkeit der frühen Sparkassen 1760-1838. Hamburg 2006, S. 30. 
3 Zitiert nach Josef Wysocki: Untersuchungen zur Wirtschaftsund Sozialgeschichte der deutschen Sparkassen im 19. Jahrhundert. Stuttgart 1980, S. 198.

4 Vgl. Thorsten Wehber: ,... die Emancipation des dritten Standes bis tief in die untern Classen vollenden". Die ethische und gesellschaftspolitische Mission der frühenSparkassen.In:GeorgFahrenschon/Johannes Wallacher (Hrsg.): Ethik und Finanzwirtschaft. Stuttgart 2015, S. 65-78. manuel Kant sahen im Menschen ein vernunftbegabtes Wesen, das prinzipiell in der Lage ist, aufgrund seiner rationalen Einsichten und gemäß seinen Interessen selbstbestimmt und selbstverantwortlich zu handeln. Damit er dies auch wirklich tun kann, müssen freilich die sozioökonomischen Verhältnisse entsprechend gestaltet sein. Viele der Zirkel und Sozietäten, in denen sich aufgeklärte Bürger, Adlige und Geistliche organisierten, widmeten sich deshalb auch der praktischen Verbesserung der wirtschaftlichen und sozialen Zustände in ihren Gemeinwesen.

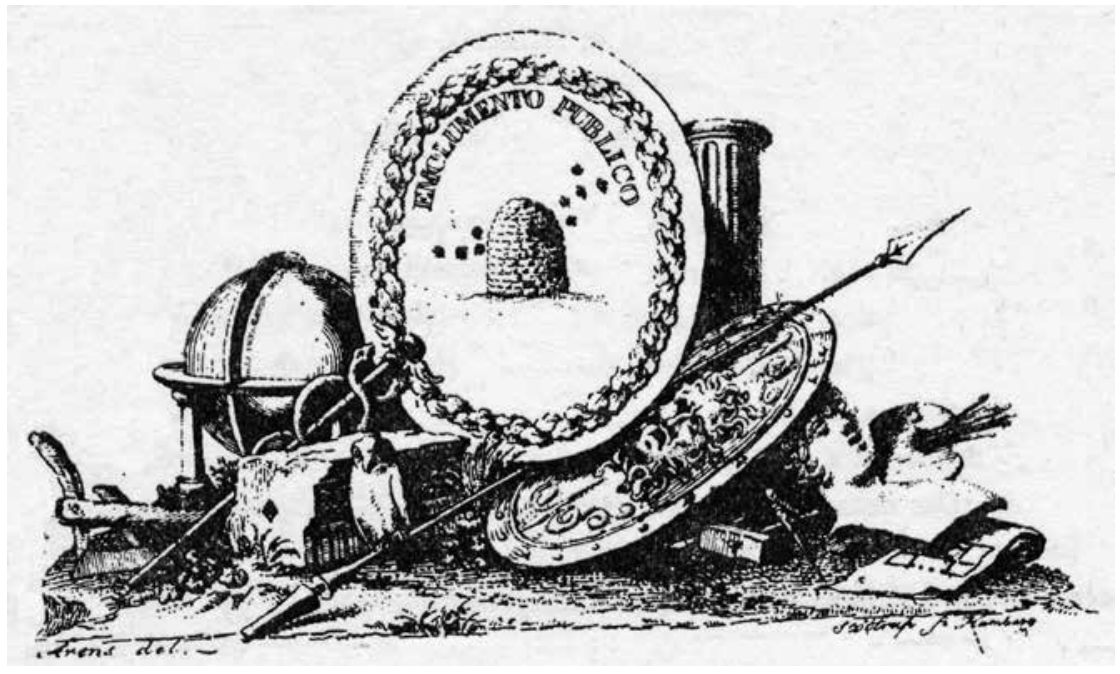

Vignette der Patriotischen Gesellschaft Hamburg. Die erste Sparkasse der Welt entstand „Emolumento Publico", zum Wohle der Allgemeinheit, 1778 in Hamburg. Viele Sparkassen verwendeten später ebenfalls den Bienenkorb und warben so für das, was er symbolisierte: Fleiß und Vorsorge. - Historisches Archiv des

Ostdeutschen Sparkassenverbandes

5 Vgl. Franklin Kopitzsch: Aufklärungssozietäten als Vermittler des Sparkassengedankens. In: Manfred Pix/Hans Pohl (Hrsg.): Invention - Innovation - Diffusion. Die Entwicklung des Spar- und Sparkassengedankens in Europa. Stuttgart 1992, S. 77-90.

6 Adolf Trende: Geschichte der deutschen Sparkassen bis zum Anfang des 20. Jahrhunderts. Stuttgart 1957, S. 53, $57 \mathrm{ff}$.

7 Kopitzsch (wie Anm. 5), S. 79 f.

8 Vgl. Thorsten Wehber: Tambora und die Sparkassen. Regionale Wirkungen einer globalen Katastrophe. In: Haus der Geschichte BadenWürttemberg (Hrsg.): Tambora. Ein Vulkan verändert Süddeutschland. UbstadtWeiher 2017, S. 85-105.
Dazu gehörte die 1765 in der Freien Reichsstadt Hamburg entstandene Gesellschaft zur Beförderung der Künste und nützlichen Gewerbe („Patriotische Gesellschaft“). Sie gründete 1778 eine „Allgemeine Versorgungs-Anstalt“, zu deren diversen Vorsorgeeinrichtungen auch eine als „ErsparungsClasse“ bezeichnete Sparkasse gehörte. Über ihren Zweck hieß es in der Satzung: „Die ErsparungsClasse dieser Versorgungs=Anstalt ist zum Nutzen geringer fleissiger Personen beyderley Geschlechts, als Dienstboten, Tagelöhner, Handarbeiter, Seeleute etc. errichtet, um ihnen Gelegenheit $\mathrm{zu}$ geben, auch bey Kleinigkeiten etwas zurückzulegen, und ihren sauer erworbenen Noth= oder Braut=Pfennig sicher zu einigen Zinsen belegen zu können, wobey man hoffet, daß sie diese ihnen verschaffte Bequemlichkeit sich zur Aufmunterung gereichen lassen mögen, um durch Fleiß und Sparsamkeit dem Staate nützlich und wichtig zu werden.“"

Diese wenigen Zeilen enthalten Grundprinzipien, die allen Sparkassen im späten 18. und frühen 19. Jahrhundert gemeinsam waren:

- Sparkassen waren Einrichtungen insbesondere für Menschen mit geringen und prekären Einkommen, ausdrücklich für Männer und Frauen.

- Sie nahmen schon kleine Geldbeträge als Einlage an und verzinsten diese.

- Sie versprachen ihren Sparern die Sicherheit des eingezahlten Kapitals, d. h. die Gewähr, dass die Ersparnisse vollständig und mitsamt den aufgelaufenen Zinsen zurückgezahlt werden.
Im damaligen, noch unterentwickelten Finanzsystem stellte die „Ersparungs-Classe“ eine Innovation dar. Erstmals gab es eine Institution, die von Verarmung bedrohten Grenzexistenzen die Möglichkeit zur individuellen finanziellen Vorsorge bot.

Der Schluss der zitierten Passage (,wobey man hoffet...") ist für das Verständnis der frühen Sparkassen nicht weniger zentral als die aufgezählten Prinzipien. Den Gründern ging es nicht nur um den unmittelbaren materiellen Nutzen, den die Einleger aus ihren Ersparnissen ziehen sollten. Fast noch wichtiger waren die erhofften Wirkungen auf den Charakter, die Wertvorstellungen und die Lebensweise der Sparer. Durch Einübung der bürgerlichen Tugend der Sparsamkeit, die sowohl Selbstbeschränkung als auch vorausschauende Planung erforderte, sollten diese zum sozialen Aufstieg befähigt werden. Die frühen Sparkassen verstanden sich somit vor allem als Erziehungsinstitutionen, welche die Emanzipation der Unterschichten und deren Integration in die entstehende bürgerliche Gesellschaft bezweckten. ${ }^{4}$

Die Gründung der „Ersparungs-Classe“ wurde durch den intensiven Gedanken- und Wissensaustausch zwischen aufgeklärten Intellektuellen sowie den vielerorts bestehenden gemeinnützigen Sozietäten weit über Hamburg hinaus bekannt. ${ }^{5}$ Dennoch blieb ihre Ausstrahlung begrenzt. Die erste am Hamburger Muster orientierte Sparkasse entstand 1786 im Herzogtum Oldenburg. 1796 folgte eine Sparkasse in Kiel, die seit 1799 über eine mit ihr verbundene „Leihkasse“ auch Kredite vergab. 1801 kam es zur Gründung von Sparkassen in Altona und Göttingen. Die Letztere ist historisch bedeutsam, weil erstmals eine Stadtgemeinde für die Einlagen bürgte. 1808 schließlich wurde in Darmstadt durch den Großherzog von Hessen die erste Sparkasse südlich des Mains errichtet. Eine Anzahl von Sparkassenprojekten in anderen deutschen Städten kam über das Planungsstadium nicht hinaus. ${ }^{6}$ Auch in der Schweiz entstanden gegen Ende des 18. Jahrhunderts Sparkassen, die möglicherweise von den Gründungen in Hamburg und Oldenburg inspiriert waren. ${ }^{7}$

Die Durchsetzung der Sparkassenidee in Deutschland gelang erst ab 1815 nach dem Sturz Kaiser Napoleons I., mit dem eine von Kriegen und tiefgreifenden politischen Veränderungen geprägte Epoche endete. Auch danach war die wirtschaftliche Lage in vielen Regionen schwierig. 1816/17 führten katastrophale Wettereignisse, verursacht durch den Ausbruch des indonesischen Vulkans Tambora, in großen Teilen West- und Süddeutschlands zu einer Hungerkrise und Massenarmut. $\mathrm{Zu}$ den sozialpolitischen Reformvorhaben, die danach in Angriff genommen wurden, gehörte auch die Gründung von Sparkassen. ${ }^{8}$ Das herausragende Beispiel dafür war die Errichtung der Württembergischen Sparkasse durch Königin Katharina Pawlowna von Württemberg im Jahr 1818. Anregungen fand die Königin nicht nur in Deutschland und der Schweiz, sondern vor allem 
auch in Großbritannien, wo sich die Sparkassen damals rasant verbreiteten. ${ }^{9}$

Das britische Vorbild beflügelte die Sparkassenbewegung auf dem Kontinent generell. Wirksam war es vermutlich auch, als 1818 in Berlin die erste Sparkasse auf preußischem Boden gegründet wurde..$^{10}$ Dabei nutzte der Berliner Magistrat die neuen Freiheiten, welche die Städteordnung von 1808 den Kommunen für die Gestaltung ihrer inneren Verhältnisse gab. Auf der Basis der kommunalen Selbstverwaltung und teilweise nach dem Muster des Berliner Instituts entstanden in den folgenden Jahren Sparkassen in allen preußischen Provinzen. ${ }^{11}$ Auch in Staaten, in denen die Städte weniger Autonomie als in Preußen besaßen, entwickelte sich die kommunale Sparkasse zum vorherrschenden Typus, so in Bayern, wo die erste Sparkasse 1821 in Nürnberg eröffnet wurde. In den Freien Städten Hamburg, Bremen, Lübeck und Frankfurt am Main sowie in Schleswig und Holstein dominierten hingegen die von privaten Geldgebern gegründeten und garantierten Sparkassen.

Von wenigen Ausnahmen abgesehen, blieben Sparkassen zunächst ein Phänomen der mittleren und großen Städte. Die Landbevölkerung konnte sie nur nutzen, wenn sie in deren Nähe lebte oder diese zum Beispiel an Markttagen besuchte. Die erste preußische Kreissparkasse wurde 1831 in Thüringen errichtet.

Eine erste - noch sehr lückenhafte - Zählung der in den Staaten des Deutschen Bundes (ohne Österreich und Herzogtum Schleswig) vorhandenen Sparkassen ergab für 1836 die Zahl von 281 Instituten. Davon bestanden unter anderem 80 in Preußen, 62 in Bayern, 32 in Württemberg und 29 in den Herzogtümern Holstein und Lauenburg. ${ }^{12}$ In Preußen widmeten staatliche Stellen den Sparkassen Mitte der 1830er Jahre erhöhte Aufmerksamkeit. Als Resultat einer landesweiten Enquete erließ König Friedrich Wilhelm III. am 12. Dezember 1838 ein „Reglement, die Einrichtung des Sparkassenwesens betreffend“. ${ }^{13}$ Darin wurden allgemeine Vorschriften für die Errichtung, den Geschäftsbetrieb und die Beaufsichtigung kommunaler Sparkassen kodifiziert. Ein zentrales Anliegen, das die Regierung mit dem Reglement verfolgte, war die Sicherheit der bei den Sparkassen angelegten Kapitalien und damit der Schutz der Sparer. Auch Bayern setzte 1843 eine „Verordnung betreffend die Grundbestimmungen der Sparkassen“ in Kraft. Diese zwang jedoch die bayerischen Sparkassen in ein sehr viel engeres rechtliches Korsett als die preußische Regulierung, die den Instituten viele Freiräume ließ.

Das Sparkassenreglement hatte einen großen Anteil daran, dass die Zahl der Sparkassen im größten deutschen Einzelstaat nach 1840 steil anstieg. 1850 gab es in Preußen bereits 234 Institute, 1860 hatte sich ihre Zahl auf 471 verdoppelt, und 1900 bestanden 1490 Sparkassen. ${ }^{14}$ Im Jahr 1900 existierten im gesamten Deutschen Reich 2.685 Sparkassen. $^{15}$

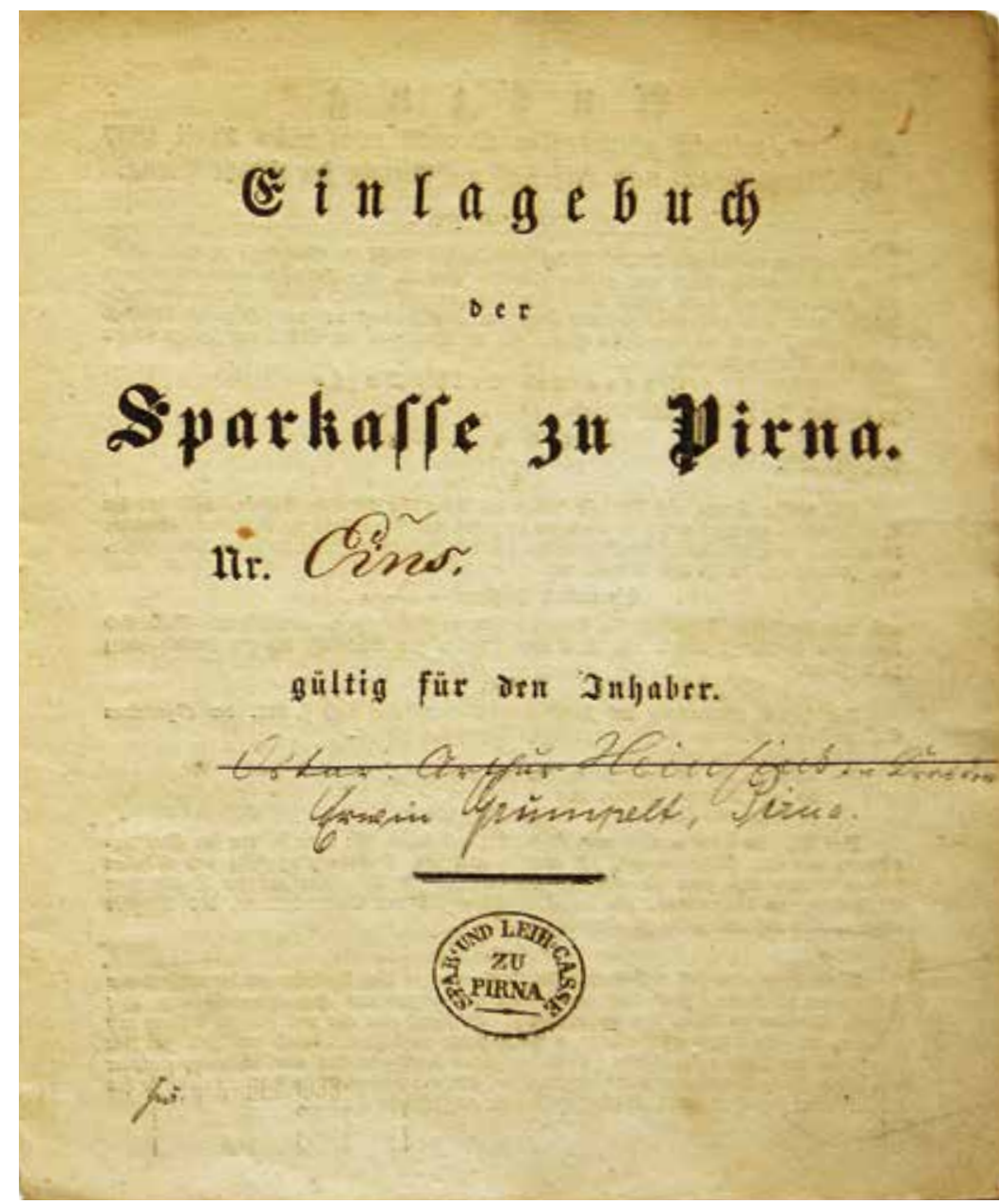

Diese Zahlen spiegeln zweifellos den großen Erfolg wider, den die Institution Sparkasse im 19. Jahrhundert hatte. Schon die Zeitgenossen stellten sich aber die Frage, ob die Sparkassen ihrem ursprünglichen Zweck gerecht wurden, die ärmeren Bevölkerungsschichten zum Sparen zu motivieren. Die Antwort darauf ist durchaus ambivalent.

Zunächst ist festzuhalten, dass die Sparkassen die „Bettelarmen“ nicht erreichten, also alle, die ihren Lebensunterhalt nicht selbst bestreiten und folglich auch nicht sparen konnten. Deren Zahl war aber gerade in der ersten Hälfte des 19. Jahrhunderts noch sehr hoch.

Anders sah es bei den „Unbemittelten“ aus, den Menschen mit einem mehr oder weniger regelmäßigen Einkommen wie Handwerksgesellen, Dienstboten und Tagelöhnern. Das war die Zielgruppe, die schon die Hamburger „Ersparungs-Classe“ von $1778 \mathrm{im}$ Blick hatte und die auch in vielen anderen zwar bei allen Sparkassen eine der größten Kundengruppen, aber in der Regel nicht die Mehrheit der Sparer. Das lag jedoch nicht - wie viele bürgerliche Gründer und Förderer der Sparkassen glaubten - an fehlender Einsicht oder am Unwillen zu sparen, sondern an der mangelnden Sparfähigkeit der unteren Einkommens- und Gesellschaftsschichten. Schätzungen gehen davon aus, dass von Sparkassensatzungen genannt wurde. Sie bildete
Sparkassenbuch der Sparkasse Pirna, Nr. 1, erste Einzahlung am 5. Januar 1838, aufgelöst am 15. August 1945

( ) Privatarchiv Grumpelt
9 Vgl. Michael Moss: Ein Penny ist sehr wenig. Die Sparbewegung im Vereinigten Königreich von der Ruthwell Bank bis zur Privatkundenbank. In: Robert Muschalla (Hrsg.): Sparen. Geschichte eienr deutschen Tugend. Berlin 2018, S. 165-176.

10 Peters (wie Anm. 2), S. 63.

11 Vgl. Paul Thomes: Die Diffusion der Sparkassen in Preußen. In: Pix/Pohl (wie Anm. 5), S. 187-205

12 Carl August Freiherr von Malchus: Die Sparcassen in Europa. Vollständiger Nachdruck der Originalausgabe von 1838 mit einem Nachwort von Manfred Pix und Josef Wysocki. Stuttgart 1994 S. 186, 191. 
Sparkassenbuch der Sparkasse Pirna, Nr. 1, erste Einzahlung am 5. Januar 1838, aufgelöst am 15. August 1945 (- Privatarchiv Grumpelt

13 Vgl. Thorsten Wehber: Das preußische Sparkassenreglement von 1838. Individuelle finanzielle Vorsorge in kommunaler Regie. In: Dieter Lindenlaub/Carsten Burhop/ Joachim Scholtyseck (Hrsg.): Schlüsselereignisse der deutschen Bankengeschichte. Stuttgart 2013, S. 90-104.

14 Heinrich Höpker: Entwicklung und heutiger Stand der Sparkassen. In: Heinrich Höpker: Die deutschen Sparkassen, ihre Entwicklung und heutige Bedeutung. Vollständiger Nachdruck der Originalausgabe von 1924 mit einer Einführung von Hans Pohl. Stuttgart 1997, S. 78-86, hier S. 80.

15 Ebenda.

16 Vgl. Wysocki (wie Anm. 3), S. 88.

17 Ebenda.

18 Vgl. Ingo Krüger: Geschichte der bayerischen Sparkassen: In: Wissenschaftsförderung der Sparkassen-Finanzgruppe e.V. (Hrsg.): Regionalgeschichte der Sparkassen-Finanzgruppe. Stuttgart 2010, S. 295-340, $31 \mathrm{f}$.

19 Wysocki (wie Anm. 3), S. 167 ff. 20 Etienne Laspeyres: Sparkassen. In: Johann Caspar Bluntschli (Hrsg.): Deutsches StaatsWörterbuch. Bd. 9. Stuttgart 1865, S. 599-608, hier S. 600.

21 Vgl. Ashauer (wie Anm. 1), S. 132, 113 ff., 134.

22 Vgl. Sandra Maß: Schulsparkassen, Kinder und Politik: Die Sparbewegungen im letzten Drittel des 19. Jahrhunderts. In: Robert Muschalla (Hrsg.): Sparen. Geschichte einer deutschen Tugend. Berlin 2018, S. 123-134.

23 Ashauer (wie Anm. 1), S. 114.

24 Ashauer (wie Anm. 1), S. 123.

25 Ashauer (wie Anm. 1), S. 119.

26 Vgl. Moss (wie Anm. 9), S. $165-178$

27 Wysocki (wie Anm. 3), S. 126 ff; Wehber (wie Anm. 13), S. 100.

Autor

Dr. Thorsten Wehber Sparkassenhistorisches Dokumentationszentrum des

Deutschen Sparkassen- und Giroverbandes Simrockstraße 4, 53113 Bonn Thorsten.Wehber@dsgv.de

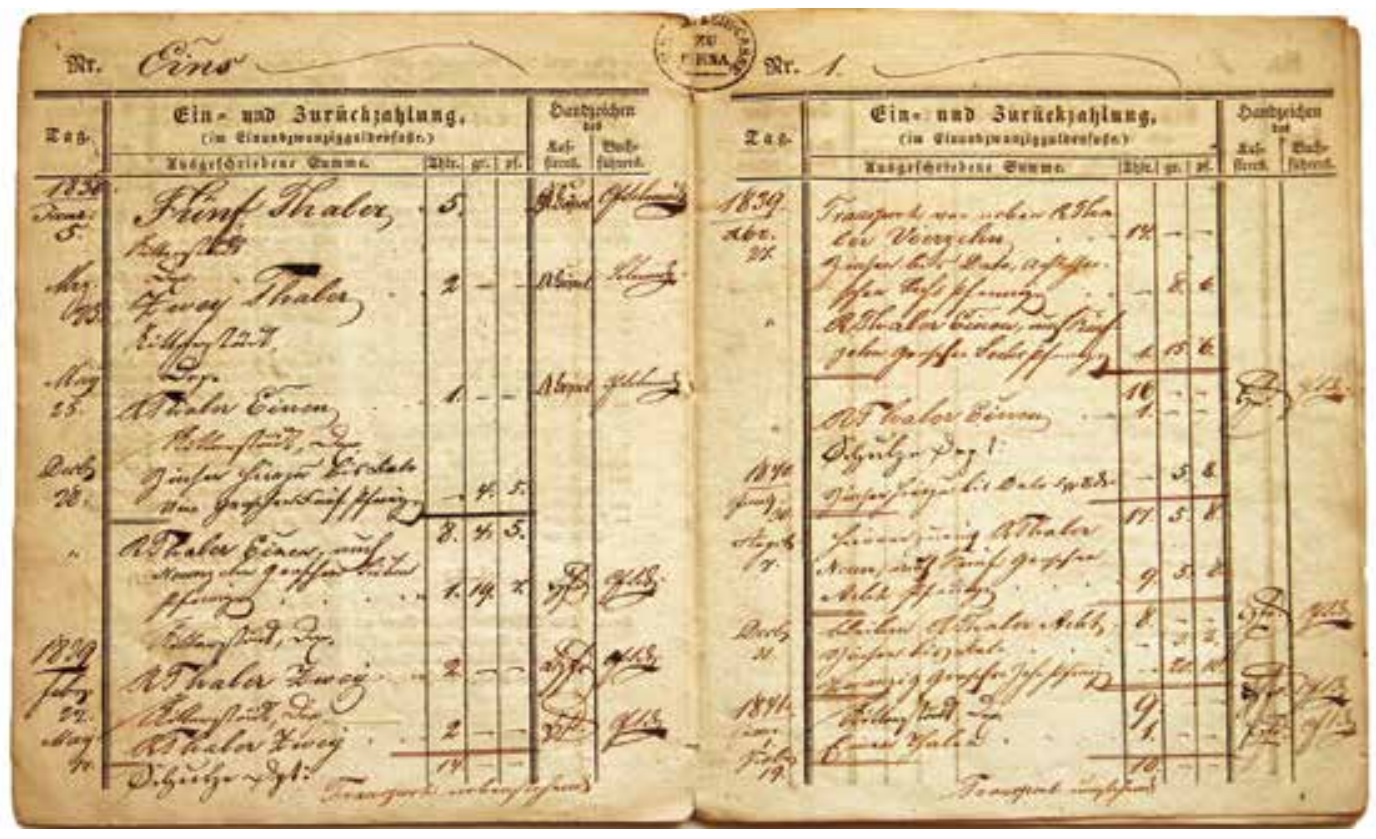

deren Gesamtheit nur „rd. 10\% die Bildung von nachhaltigen Ersparnissen gelang “. ${ }^{16}$ Dennoch trugen diese Sparer einen Anteil von 20 bis 30 Prozent zum Mittelaufkommen der Sparkassen bei. ${ }^{17}$

Das Gros der Kunden wie auch der Einlagen stammte allerdings aus dem sogenannten Mittelstand, also der breiten Schicht von selbständigen Handels- und Gewerbetreibenden, Landwirten, Beamten, Lehrern, Geistlichen etc. Dies galt bereits für die Sparkassen zu Beginn des 19. Jahrhunderts. Deren Satzungen sollten zwar häufig wohlhabendere Kunden fernhalten, indem sie die soziale Zielgruppe eng definierten und die Höhe der Einlagen pro Sparer stark begrenzten. Solche Vorkehrungen hatten aber in der Regel wenig Erfolg.

Verfechter eines Ideals, das die Sparkassen primär als Instrumente der Sozialpolitik betrachtete, mochten die Öffnung für die Mittelschicht als Abkehr von den ursprünglichen Prinzipien verurteilen. Es ist jedoch zweifelhaft, ob die Sparkassen sich so erfolgreich entwickelt hätten, wenn sie eine Einrichtung für die ärmeren Bevölkerungsschichten geblieben wären. Bayern, das seine Institute durch die Verordnung von 1843 in diese Nische zwang, erkaufte das mit einer relativen Stagnation des Sparkassenwesens. ${ }^{18}$

Die Diskussionen um die Aufgaben und das Selbstverständnis der Sparkassen, die insbesondere nach der Reichsgründung 1871 geführt wurden ${ }^{19}$, hatten durchaus Folgen. Einerseits blieb es zwar dabei, dass die Sparkassen Einrichtungen für alle Bevölkerungsschichten waren. Andererseits bemühten sich viele von ihnen, gerade der Arbeiterschaft das Sparen zu erleichtern. Dahinter standen auch politische Motive, galt doch die „Sparkasseneinlage“ als „ein Damm gegen kommunistische Gelüste und revolutionäre Gedanken, wie sie in denen auftauchen, welche nichts zu verlieren haben.“" ${ }^{20}$ Es wurden beispielsweise die Öffnungszeiten ausgeweitet, Zweig- und Annahmestellen außerhalb der Stadtzentren und in den Dörfern eröffnet und die Mindesteinzahlung herabgesetzt. $^{21}$
In diesen Zusammenhang gehören auch viele Maßnahmen, die das „Kleinsparen“, d. h. das Sparen schon kleinster Geldbeträge, fördern sollten. Die Schul- und Jugendsparkassenbewegung erhielt in dieser Zeit großen Auftrieb ${ }^{22}$ und ebenso die sogenannten „Pfennigsparkassen“. Vielerorts konnten die Menschen in Ladengeschäften Sparmarken erwerben, die sie, war eine bestimmte Summe erreicht, zur Eröffnung eines Sparkontos oder zur Einzahlung auf ein bestehendes Konto nutzen konnten. Nach 1900 wurden für diesen Zweck auch Sparautomaten aufgestellt. Sparkassen begannen nun auch, verschlossene „Heimsparbüchsen“ auszugeben, mit denen ihre Kunden zuhause sparten - der Schlüssel blieb bei der Sparkasse. Manche Institute führten zudem einen „Abholdienst“ ein, bei dem Sparkassenmitarbeiter die Ersparnisse direkt in der Wohnung des Kunden einsammelten.

Um die Wende zum 20. Jahrhundert hatte sich Deutschland zu einem Land der Sparkassen und der Sparerinnen und Sparer entwickelt. Bei 9.127 Sparkassen und Sparkassenfilialen konnten die Menschen 1905 ihre Ersparnisse einzahlen. ${ }^{23}$ Über 17 Millionen Sparkassenbücher waren ausgegeben. ${ }^{24}$ Das bedeutete, dass mehr als ein Viertel der rund 60 Millionen Deutschen ein Sparkonto bei einer Sparkasse besaß. Der Einlagenbestand belief sich auf etwa 12,7 Milliarden Mark. ${ }^{25}$

Die deutschen Sparkassen waren dadurch auch zu einem wichtigen volkswirtschaftlichen Faktor geworden. Anders als in Großbritannien ${ }^{26}$ beschränkten sie sich nicht darauf, die Spareinlagen in Staatspapieren anzulegen. Vielmehr hatten sie in der Regel ein breit gefächertes Kreditgeschäft aufgebaut. Es umfasste Darlehen an Privathaushalte, Handel, Handwerk, Industrie und Landwirtschaft ebenso wie Kredite an ihre Träger, die Kommunen. ${ }^{27}$ Letztere profitierten auch von Überschüssen der Sparkassen, die beispielsweise dem Schul- und Gesundheitswesen und der sozialen Fürsorge zugutekamen. 